\title{
Group Attention Control for Communication Robots with Wizard of OZ Approach
}

Masahiro Shiomi, ${ }^{1,2}$ Takayuki Kanda, ${ }^{1}$ Satoshi Koizumi, ${ }^{1,2}$ Hiroshi Ishiguro, ${ }^{1,2}$ Norihiro Hagita ${ }^{1}$

\author{
1 ATR IRC Laboratories \\ 2 Osaka University \\ Kyoto 619-0288 \\ Japan \\ Osaka 565-0871 \\ Japan \\ m-shiomi@atr.jp kanda@atr.jp satoshi@atr.jp ishiguro@atr.jp hagita@atr.jp
}

\begin{abstract}
This paper describes a group attention control (GAC) system that enables a communication robot to simultaneously interact with many people. GAC is based on controlling social situations and indicating explicit control to unify all purposes of attention. We implemented a semi-autonomous GAC system into a communication robot that guides visitors to exhibits in a science museum and engages in free-play interactions with them. The GAC system's effectiveness was demonstrated in a two-week experiment in the museum. We believe these results will allow us to develop interactive humanoid robots that can interact effectively with groups of people.
\end{abstract}

\section{Categories and Subject Descriptors}

H.5.2 [Information Interfaces and Presentation]: User Interfaces - Interaction styles, I.2.9 [Artificial Intelligence]: Robotics

\section{General Terms}

Design, Experimentation, Human Factors

\section{Keywords}

Commutation robot, Group attention control, Field trial, Humanrobot interaction, Science museum robot

\section{INTRODUCTION}

The development of robots is entering a new stage whose focus is placed on interaction with people in daily environments. The concept of communication robot continues to rapidly evolve. A robot will act as a peer providing mental, communication, and physical support. For example, pet-type robots such as AIBO [1] and PARO [2] have been developed to provide mental care. Humanoid robots have also been developed for the research of Human-Robot Interaction. These robots investigate how humanlike body movements or facial expressions contribute to interaction with people [3-5]. In addition, robots have operated in

Permission to make digital or hard copies of all or part of this work for personal or classroom use is granted without fee provided that copies are not made or distributed for profit or commercial advantage and that copies bear this notice and the full citation on the first page. To copy otherwise, or republish, to post on servers or to redistribute to lists, requires prior specific permission and/or a fee.

Conference'04, Month 1-2, 2004, City, State, Country.

Copyright 2004 ACM 1-58113-000-0/00/0004 ...\$5.00 such daily environments as elementary schools and museums [69].

These robots, however, were not designed to simultaneously interact with many people. Figure 1 shows an interaction scene between a robot and a crowd in a museum. In such environments, people surround a communication robot when it performs such tasks as greeting, explaining, or guiding. We believe that knowing how to create a communication robot that interacts with many people in daily environments is important.

Therefore, we propose a group attention control (GAC) system for communication robots that allows the robot to interact with many people by addressing why people pay attention to robots. People make various demands of robots. For example, if half of the people want to play with the robot and the other half want to listen to its explanations, their demands will not be satisfied due to the conflict. Instead, if they behaved in a time-sharing manner, all demands will be satisfied. The GAC system tries to control everyone's purpose of attention to avoid this conflict.

However, in the development process a key difficulty exists for the interaction of such a communication robot with many people. It must be placed in a real field; otherwise, we cannot reproduce similar situations. For example, we tried to reproduce real field settings in our laboratory with several researchers, but we failed because people in the field do not behave as predicted. This trend is particularly obvious if the number of people is large or diverse, including children, adults, and senior citizens, and if there are many objects in the environment. Such situations highly activate interaction among people, resulting in an excessively chaotic situation.

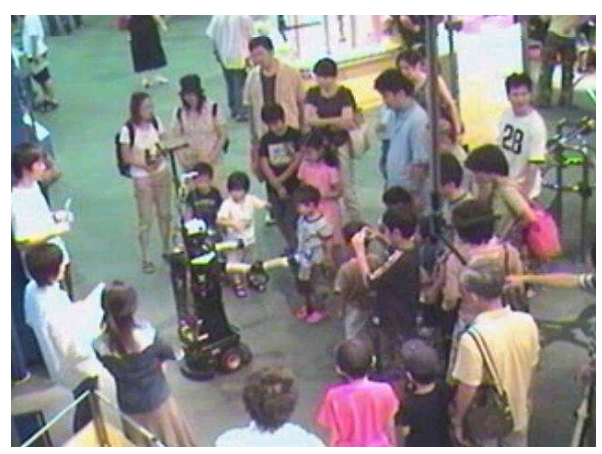

Figure 1 Robot simultaneously interacting with many people 
We adopted an approach with a semi-autonomous system to solve this problem and tested the GAC system with the Wizard of $\mathrm{Oz}$ (WOZ) method. Previous works have also demonstrated the WOZ method's usefulness for studying human reactions in human-robot interaction [10, 11]. Our focus concentrates on the behaviorgenerating mechanism in the GAC system. In the development process, two issues must be addressed. The first is the sensing part We need to retrieve necessary information from the environment to create autonomous robots. The other is a behavior-generating mechanism manipulated by the robot to decide its action using sensed information. We designed a behavior-generating mechanism for GAC, tested it, and improved it in a real field.

In this paper, we describe the effects of a GAC system in interaction between a robot and a group. We implemented the GAC system on a communication robot that acts as an exhibit guide and engages in free-play interactions in a science museum. The system is currently semi-controlled using the Wizard of $\mathrm{Oz}$ method.

\section{ATTENTION CONTROL}

\subsection{Group Attention Control}

We concentrated on the focus and purpose of attention to control group attention. Focus means the direction of attention, and purpose explains why people are aiming their attention in that direction. Based on these ideas, we made a model of group attention for human-robot interaction (Figure 2). $\mathrm{R}$ denotes the robot, $\mathrm{H}_{\mathrm{i}} \ldots \mathrm{H}_{\mathrm{N}}$ denotes humans interacting with the robot, $\mathrm{F}_{\mathrm{i}} \ldots \mathrm{F}_{\mathrm{N}}$ denotes focus of attention $\mathrm{H}_{\mathrm{i}}$, and $\mathrm{P}_{\mathrm{i}} \ldots \mathrm{P}_{\mathrm{N}}$ denotes the purpose of $\mathrm{H}_{\mathrm{i}}$.

There are several research works in the field of Human-Robot Interaction on focus of attention [3-8]. For example, a museum robot called visitor names for attention control. In contrast, little research has been done to coordinate the attention of groups. We believe that purpose of attention becomes more important when a robot interacts with many people: if the purpose of many people's attention is different, the robot have difficulty simultaneously satisfying everyone.

However, if the robot can coordinate all purposes through its behavior, it can satisfy various purposes in time by performing various tasks. For example, in a science museum, the robot might talk about an exhibit, move around other exhibits, and let visitors experience its own novel technology by allowing and encouraging interaction with it.

\subsection{Design for Group Attention Control}

We established two principal design guidelines for GAC based on the proposed model.

\subsubsection{Creating a "cooperative situation"}

We define social situation as a state in which people are aware of and notice each other. In such states, people might need to adjust their own purpose to avoid conflict with others. In other words, by creating a social situation, each $\mathrm{P}_{\mathrm{i}}$ is adjusted to become identical to a particular one.

When creating a social situation, we believe that a robot should control people's physical positions because they will lose interest in the robot if they cannot interact with it for an extended period or are too far from it. Thus, for a social situation, a robot must make such statements as "Please stand side by side in front of me" or "Move closer."

In addition, robots should also use such personal information as age or name to encourage parents or adults to give priority to children. It seems reasonable that a robot should engage children in museums visited by many families.

\subsubsection{Explicitly indicating context}

We focused on the process of indicating either implicit or explicit contexts. Since people usually prefer to implicitly exchange context, the behavior of interactive robots is conventionally designed with an implicit arrangement of context. For example, when starting to chat with visitors, a robot [3] shakes hands instead of saying, "Now, our greeting is done, so let's start talking." Such explicit indication of context is unnatural in daily situations.

On the contrary, when a robot has multiple tasks and is interacting with a large number of people, explicit context changes are important. Robot behavior strongly reflects the meaning of a task when the robot is doing that task. Thus, unnatural behavior sequences may result when the robot changes tasks. For example, in a museum context, visitors might be nonplussed if the robot says "please hug me" after explaining how to play with an exhibit. Indicating explicit context is also effective for avoiding human misinterpretations. In other words, a robot must be able to unify everyone's purpose by explaining the next context before changing to it.

\section{SYSTEM CONFIGURATION}

\subsection{System Overview}

The purpose of our developed system is to create a robot that simultaneously interacts with many people by controlling group attention and provides such services as explaining exhibits and playing with visitors. The robot's services include entertaining visitors and explaining exhibits because past research indicated their effectiveness [8].

Figure 3 shows a map of the Osaka Science Museum, camera locations, and the robot (Figure 3A). The robot has two systems: an autonomous system for interaction with people and a semiautonomous system for controlling group attention and navigation. The latter system is managed by an operator using a remote operation system that consists of a personal computer, a database, five cameras, and four monitors that display camera images. The remote operation system was installed in the museum, as shown in Figure 3B.

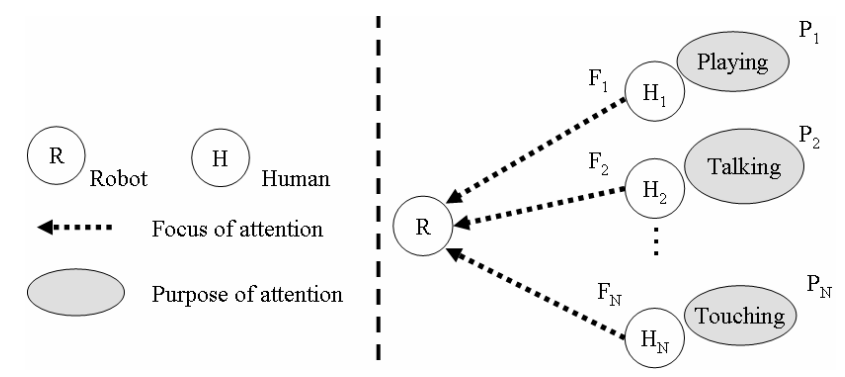

Figure 2 Proposed model of group attention 


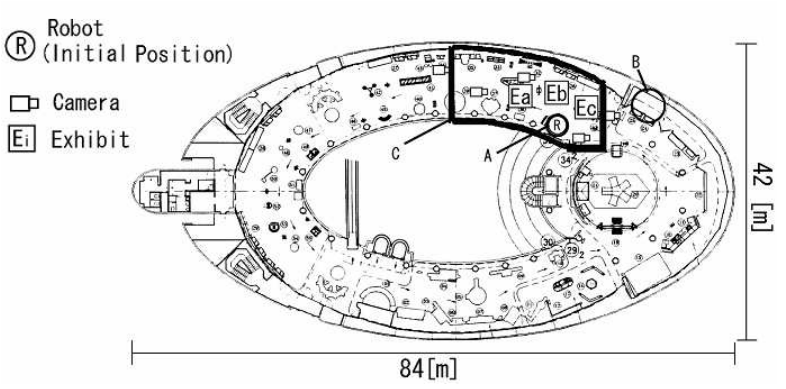

Figure 3 Map of Osaka Science Museum

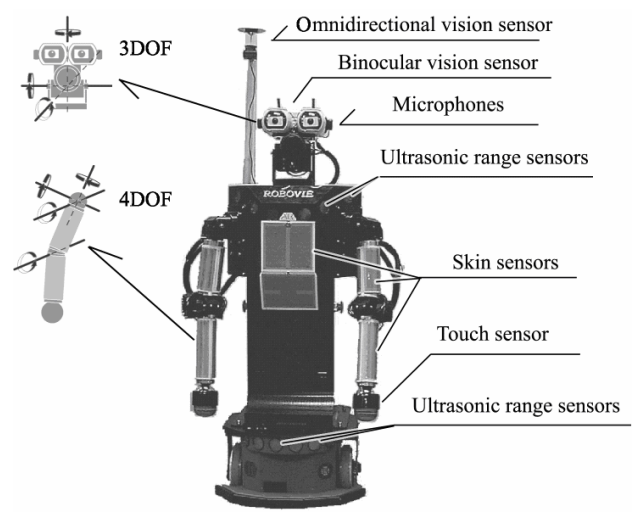

Figure 4 Robovie

The robot has three basic tasks: playing, moving, and explaining. Its work area is represented by Figure $3 \mathrm{C}$. The robot explained the three exhibits represented by $\mathrm{Ea}, \mathrm{Eb}$, and $\mathrm{Ec}$.

\subsection{Hardware Configuration of "Robovie"}

Figure 4 shows "Robovie," an interactive humanoid robot characterized by its human-like physical expressions and its various sensors. We used humanoid robots because a human-like body is useful for naturally controlling people's attention [3]. A human-like body consists of a head, a pair of eyes, and two arms. When combined, these parts can generate the complex body movements required for communication. We decided on a robot $120 \mathrm{~cm}$ tall to decrease the risk of scaring children. Its diameter was $40 \mathrm{~cm}$. The robot has two 4 degrees of freedom in its arms, 3 degrees of freedom in its head, and a mobile platform. It can synthesize and produce voice by a speaker.

\subsection{Software Configuration of "Robovie"}

In this section, we describe Robovie's software architecture details. Figure 5 shows the entire robot system with the GAC system implemented. The upper square represents the GAC system, and the lower represents the existing robot system. The operator controlled the part of the robot system represented by gray.

\subsubsection{Robot system}

The following is the flow of the existing robot system. Sensor modules recognize such information about the people around the robot as presence, distance, speech, and so on. The behavior controller executes a behavior based on sensor information and the Episode rules, which govern the order of execution. The actuator modules then actuate the robot to interact with people. Reactive behaviors include avoidance and gazing at a touched part of its body, as well as such patient behavior as playing by itself. These reactive behaviors are controlled based on robot sensor information from tactile, vision, and distance sensors, etc.

\subsubsection{GAC system}

The GAC system chooses a behavior to create a social situation and indicates an explicit context. It includes behaviors (Figure $5 \mathrm{~A}$ ), rules (Figure 5B), recognition functions (Figure 5C), and the behavior controller (Figure 5D).

GAC's recognition functions estimate the position and height of people for interaction. GAC's behavior controller chooses a behavior for GAC based on information from its recognition functions and rules. The chosen behavior is executed when the current behavior is finished.

\subsubsection{Remote Operation}

In this research, an operator controlled the recognition functions (Figure 5C), the behavior controller (Figure 5D), and the robot wheels, as represented by gray. Camera images are used by the operator for controlling such components. On the other hand, sound data surrounding the robot was omitted in remote operations because current technology cannot recognize speech in a crowded environment.

The operator chooses a behavior for GAC when its rules are satisfied. The chosen behavior is executed when the current behavior is finished. The timing of choosing the behavior does not affect the timing of the behavior execution (Figure 6). In contrast, the robot wheels are immediately controlled by the operator. For future work, we recorded all camera images and logs of operation data to develop an autonomous GAC system.

\subsubsection{Tasks}

The robot had four tasks: the GAC task and three basic tasks that consisted of playing, moving, and explaining. The GAC task starts when the robot's basic tasks and the GAC rules are fulfilled. In executing a task, the robot only performs behavior that belongs to each task. Exhibits are explained in the order of "Ea-Eb-Ec" or "Ec-Eb-Ea." The basic task's order is as follows:

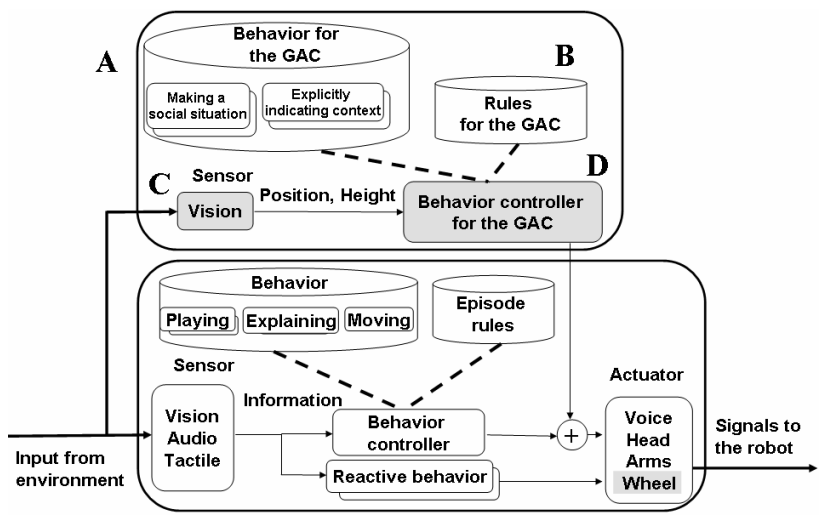

Figure 5 Robovie control system 
Table 1 GAC Behaviors for making social situation.

\begin{tabular}{|l|l|}
\hline Uttered by robot & Rules for controlling behaviors \\
\hline "Please stand side by side in front of me." & When visitors are not standing in front of robot \\
\hline "Please move back a little." & When distance between robot and nearest person is less than about $50 \mathrm{~cm}$ \\
\hline "Come closer." & When distance between robot and farthest person is over $3 \mathrm{~m}$ \\
\hline "Please bring the child closer." & When a child shorter than the robot is present \\
\hline
\end{tabular}

Table 2 GAC Behaviors for indicating of explicit context

\begin{tabular}{|l|l|}
\hline Uttered by robot & Rules for controlling behaviors \\
\hline "Hi everyone, let's have some fun!" & When next task is entertaining multiple people \\
\hline "I am taking you to an exhibit, so please follow me." & When next task is guiding \\
\hline "I am tired, goodbye!" & When robot moves to home position \\
\hline "Now, let me tell you about this exhibit." & When next task is explaining \\
\hline
\end{tabular}

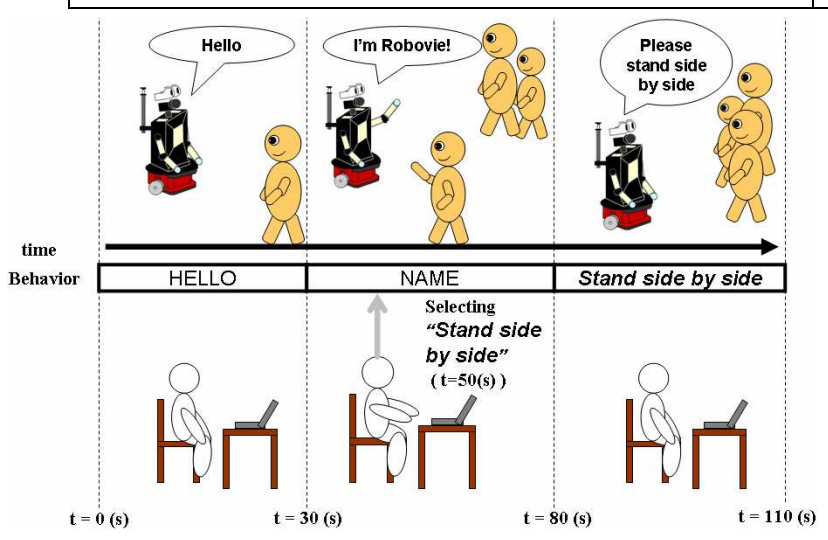

Figure 6 Example of behavior transition

(1) Freely interact with visitors for about three minutes.

(2) Guide visitors to exhibits.

(3) Explain exhibits to visitors.

(4) Repeat tasks (1) through (3) until all exhibits have been explained.

(5) The robot returns to its home position and rests for a few minutes.

\section{4 "Robovie" Behavior}

\subsubsection{Creating a cooperative situation}

Table 1 shows the behavior for creating social situations. They include adjusting the distance between the robot and visitors and interacting with children more than adults. The robot seemed to control the attention of adults by grabbing the attention of children because many families visited the experimental environment. These behaviors were selected by the operator as the robot's next behavior after satisfying the rules in Table 1 . If a situation did not change even though the robot repeated the same behavior to create the same social situation, the robot performed the next behavior of the current task.

\subsubsection{Explicitly indicating context}

Table 2 shows behavior for indicating explicit context and the rules for selecting these behaviors, which were used to indicate explicit context to visitors when the robot changed tasks. The operator selected these behaviors as well as behaviors for creating social situations.

\subsubsection{Playing}

We previously implemented such fundamental behavior for engaging in childlike behavior as handshaking, hugging, and the "rock, scissors, paper" game [3]. Moreover, the robot has such reactive behaviors as avoidance and gazing at a touched part of its body as well as such patient behavior as playing by itself. These behaviors are automatically selected based on the episode rules and robot sensor information harvested from tactile, vision, and distance sensors, etc.

\subsubsection{Moving}

We implemented behaviors for guiding visitors to the three exhibits. The robot discussed each exhibit or the museum during the guiding behavior and then began explaining again after it finished guiding.

In previous work, the robots had robust moving functions using ceiling cameras. But in this research an operator controlled the robot's speed and direction. So we easily achieved safer moving functions than an autonomous system because we had already developed the software operation. These behaviors were automatically selected based on the episode rules and robot sensor information from tactile and distance sensors. In addition, reactive behaviors were performed autonomously.

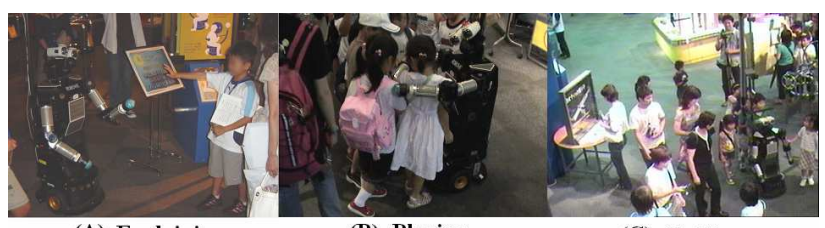

(A) Explaining

(B) Playing

(C) Guiding

Figure 7 Interaction scenes at exhibits 


\subsubsection{Explaining}

We implemented behavior for explaining and recommending the three exhibits to visitors. The robot explained the exhibits and then recommended playing with them. These behaviors were selected based on the robot's sensor information as well as freeplay interaction.

\section{Exhibition and Visitor Behavior}

In this section, we describe the exhibition details to investigate how visitors interact with the robot.

\subsection{Exhibition}

\subsubsection{In the science museum exhibition}

First, we investigated visitor interaction with the robot without the GAC system in the museum. In the exhibition, visitors freely interacted with the robot for a two-week period. For analysis, we recorded images of interaction scenes between visitors and the robot with five ceiling cameras and one digital video camera.

In the exhibition the robot executed the three basic tasks in the order of explaining, playing, and moving (Figure 7). Thus, the robot only used behaviors described in Sections 3.4.3 to 3.4.5

\subsubsection{Interaction in the museum}

1) Free-play interaction

The robot performed behaviors such as handshaking and hugging when visitors were present (Figure 7B). In crowded situations, a few children mainly interacted simultaneously with the robot in turns. Also, passers-by often approached the robot and gradually gathered around it when the robot performed free-play interaction, even if nobody had been around it prior to its playing alone. Adults generally watched the robot from a distance.

\section{2) Explaining the exhibits}

Visitors sometimes played with exhibits while listening to explanations from the robot (Figure 7A). For example, the robot might say, "These pendulums swing at different speeds. Why don't you try them?" Thus, visitors tried to swing them. On the other hand, when the robot changed to exhibit explanation from free-play interaction, some children ignored the explanation and continued to touch them.

3) Moving

When the robot started to move to a different place, approximately half of the visitors followed it, while others went off in other directions. Almost all of the followed visitors obediently moved with the robot (Figure 7C).

\section{Experiment}

We did experiments to verify the GAC system's effectiveness under the two operating conditions. The following are the details of the experimental settings:

\section{Basic Settings}

The experiment period was two weeks. We randomly changed conditions between mornings and afternoons. In the experiments, visitors could freely interact with the robot.

\section{Conditions}

\section{1) GAC condition}

The GAC system was installed on the robot. In other words, the robot performed every behavior introduced in Section 3.4. The operator controlled robot behaviors based on GAC rules. In addition, the operator controlled the speed and direction of the robot.

\section{2) Non-GAC condition}

The GAC system was not installed on the robot. In other words, the robot preformed every behavior introduced in Sections 3.4.3 to 3.4.5. The operator only controlled the speed and direction of the robot.

\section{Subjects}

Subjects were visitors who experienced exhibit explanations, guidance, or free-play interaction. After interaction ended, we asked them to fill out a questionnaire. Even children were asked to fill out questionnaires if they understood the meaning or after parents explained the questionnaire.

\section{Measurement}

To evaluate the GAC system, we prepared a questionnaire in which subjects rated items on a scale of 1 to 7 , where 7 is the most positive. The following are the questionnaire items:

\section{1) Evaluating effectiveness of creating a social situation}

We prepared three items for evaluating the effectiveness of creating a social situation. In the GAC condition, the robot tries to create a social situation by controlling the distance between itself and people or between the people. We expected more people to interact with the robot after creating a social situation than a nonGAC condition. The following are the items:

(1) Crowdedness: Was it crowded around the robot?

(2) Approachability: How approachable was the robot?

(3) Opportunity: Were there plenty of opportunities to interact with the robot?

In addition, to investigate crowdedness around the robot, we measured the number of visitors and the time spent watching the robot for more than one second within five meters of the robot. These values were measured using images from the five cameras. The measurement period was ten minutes after the start of each trial.

\section{2) Evaluating effectiveness of explicitly indicating context}

We prepared four items to evaluate the effectiveness of indicating explicit context. We expected more people to easily understand the robot's intention and exhibit explanations when an explicit context is indicated. The items include the following:

(1) Explanation: How well did you understand the robot's explanations?

(2) Naturalness: How natural was the relation between the robot's actions?

(3) Intention: How well did you understand the robot's intentions? 
(4) Interest: How much did the robot increase your interest in the exhibits?

\section{3) Investigating impressions of the robot}

We prepared one item to investigate impressions of the robot under the two conditions.

(1) Impression: What did you think about the presence of robots in the science museum?

\subsection{Results}

We received 120 questionnaires from visitors for both operating conditions. Figure 8 shows the results and the averages of the items to evaluate the effectiveness of creating a social situation. The t-test results of "Crowdedness" showed a significant difference under the GAC condition $(\mathrm{p}<.05)$, which visitors felt was less crowded than the no-GAC condition.

Figure 9 shows the total number of visitors and the average time they stayed. This time average showed no significant differences between conditions $(\mathrm{p}=.14)$. The total number did not significantly differ between conditions $(p=.54)$. These results indicate that the crowdedness of the GAC condition equals the crowdedness of the no-GAC condition.

The above two results indicate that better control of social situations solved the crowded situation around the robot. But "Approachability" and "Opportunity" results did not show a significant difference between conditions

Figure 10 shows the results and their averages for the items that evaluated the effectiveness of indicating explicit context. These results suggest that indicating explicit communication control might attract visitors to the robot because "Explanation" and "Naturalness" results showed a significant difference under the GAC condition $(p<.05)$. Moreover, "Intention" results showed a significant trend under the GAC condition $(\mathrm{p}<.1)$. On the other hand, "Interest" results did not show a significant difference between conditions.

Figure 11 shows the results of the item to investigate impressions of the robot. In the GAC condition, visitors evaluated the robot higher than the no-GAC condition $(\mathrm{p}<.05)$. In addition, results indicate that visitors had good impressions of the robot for each condition.

\section{DISCUSSION}

\subsection{Effectiveness of GAC}

We verified the GAC system's effectiveness through experiments under two operating conditions during the two-week period. Questionnaire results showed that most visitors evaluated the GAC system robots higher than without the GAC system. Here, we discuss how the GAC system contributed to interaction between the robot and visitors by introducing the details of the questionnaire results and some scenes of visitor interaction as a case study.

\section{1) Solving crowdedness situation around the robot}

In the GAC condition, visitors felt less crowdedness than the noGAC condition, although measured crowdedness showed no significant difference between the two conditions. The reason for this difference is that crowdedness around the robot was decreased by creating a social situation, as we expected. The robot adjusted the distance between itself and visitors when trying to create a social situation.

\section{2) Understanding the robot's behavior}

In the GAC condition, visitors understood the robot's explanations more than the no-GAC condition, although the amount of speech and the number of times were approximately the same between the two conditions. In fact, questionnaire results showed significant differences in "Explanation." In addition, they showed a significant trend in "Naturalness" and "Intention." Visitors easily understood the robot's behavior by indicating explicit context.

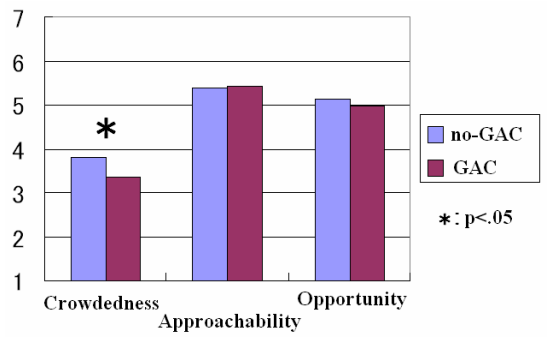

Figure 8 Questionnaire results for creating a cooperative situation

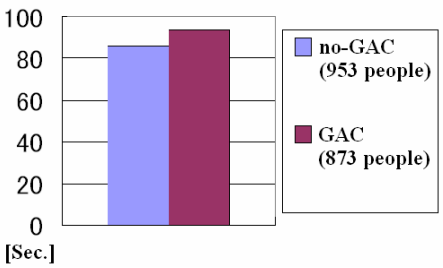

Figure 9 Number of visitors around robot and staying times, indicating that objective crowdedness has no significant difference between the two conditions.

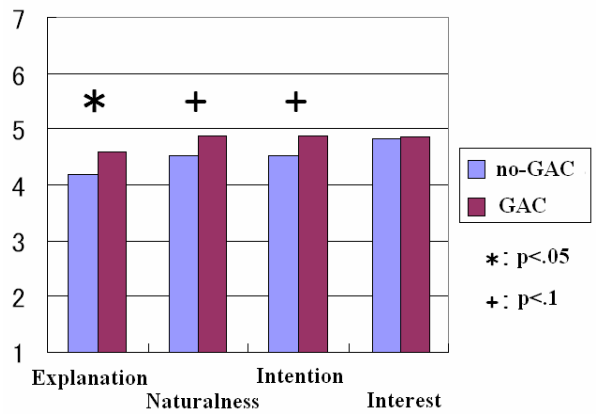

Figure 10 Questionnaire results for explicitly indicating context

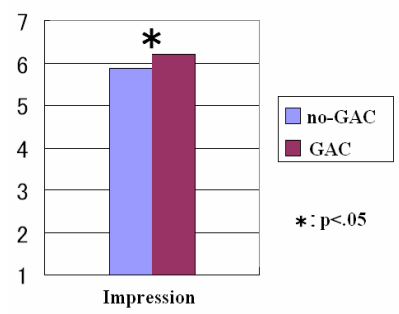

Figure 11 Questionnaire results for robot's impression 


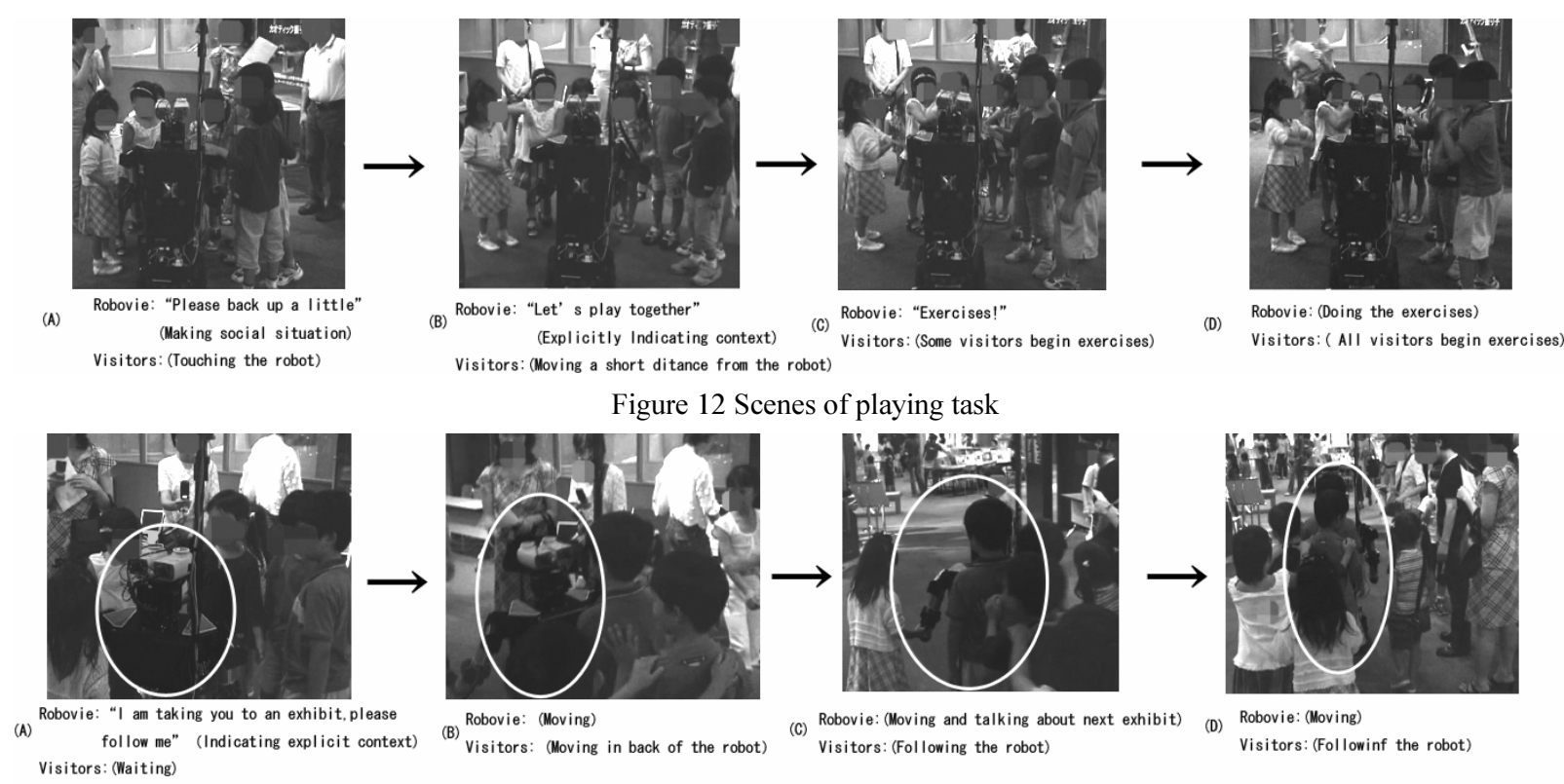
Visitors: (Waiting)

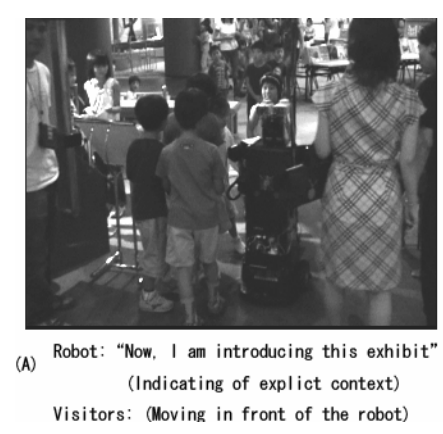

Figure 13 Scenes of moving task
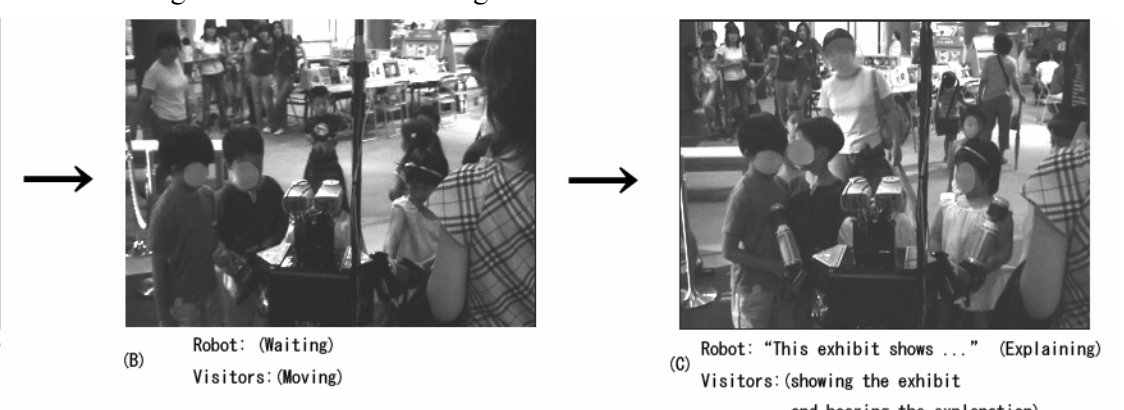

Figure 14 Scenes of explaining task

\section{3) Interesting interaction scenes in the GAC condition}

We observed some interesting scenes only under the GAC condition that demonstrate the GAC system's essential importance as well as the questionnaire results.

Figure 12 shows a playing task scene of children with the robot under the GAC condition. In Figures $12 \mathrm{~A}$ and $\mathrm{B}$, children are standing in front of the robot, and they moved away from the robot because it created a social situation. When the children on the left side mimicked the robot (Figure 12C), the children on the right side also mimicked the robot after looking at the other children (Figure 12D).

Figure 13 shows a moving task scene under the GAC condition. The robot is circled. The children are walking in a line behind it when the robot started to move to the next exhibit. Table 3 shows a conversation between the robot and visitors in playing and moving tasks. We suggest that either some social relationships were established between the children through interaction with the robot or a social situation was created.

Figure 14 shows an explaining task scene under the GAC condition. The children are anticipating the behavior of the robot and waiting for its explanation after the robot indicated the explicit context. The children are also predicting the robot's next behavior. Moreover, their parents are standing behind the children and waiting for the robot to explain. These scenes demonstrate that the GAC system robot interacted with many visitors (e.g., with multiple families) in large-scale daily environments. We believe that such scenes indicate the potential of a communication robot in daily environments.

Table 3 Example of conversation

\begin{tabular}{|l|l|}
\hline & Utterance or Action \\
\hline Robovie & Please bring the child closer. \\
\hline Parents & (They moved behind the children). \\
\hline Children & Nice to meet you, Robovie. \\
\hline Robovie & Hi everyone, let's have some fun! \\
\hline Robovie & Let's play 'rock, scissors, paper'! \\
\hline & $\ldots \ldots . .$. \\
\hline Robovie & I am taking you to an exhibit, please follow me. \\
\hline Child-A & Look! Robovie said "Please follow me." \\
\hline Child-B & Really? \\
\hline Robovie & (Robovie starts moving to an exhibit). \\
\hline Children & (They talked about Robovie while following). \\
\hline
\end{tabular}




\subsection{Influence of remote operation}

Since the robot was controlled by an operator in this research, the effects of remote operation must be examined as well as the GAC system.

The operator selected robot behaviors based on GAC rules. The provided information for the operator was only visual. Thus, the operator was not affected by such audio information as the voices of visitors.

We designed the robots so that the selected behavior becomes its next behavior because the timing of the robot selection behavior differs each time. Robot interactivity depends on an operator if the selected behavior is executed immediately after the operator selects the behavior. Namely, the timing of the selected behavior's start is identical, even if the timing of the selection behavior is different.

Furthermore, robot speed and direction were controlled by the operator in each condition. Therefore, the difference of the effects of remote operation is slight.

Therefore, the effects of remote operation were limited in the area represented by Tables 1 and 2 . The effects of remote operation were not affected by the audio information of visitors, and the interactivity of the robot did not depend on the operator.

\subsection{Implementing an autonomous GAC system}

Here we discuss the requirements for developing an autonomous GAC system. The robots must detect people's positions and identify them to autonomously control group attention because GAC was achieved using robot behaviors based on visitors' information.

To detect each person's position, we suggest floor sensors in crowded situations, such as the scenes in our experiment, since they can collect high-resolution data and are robust to occlusion.

For identification, RFID technology has advantages over the above sensors because it enables robots to easily recognize people and objects in an environment $[6,8]$. Robots can gather information about each person using person identification.

We believe that utilizing the above sensors in conjunction with robots will enable them to more effectively control group attention. It is also important to estimate a person's attention model [12]

\section{CONCLUSION}

We developed a GAC system that enables a robot to simultaneously interact with a group of people. It controls social situations and explicitly indicates contexts to unify everyone's purpose of attention. We installed the GAC system on a communication robot and verified its effectiveness in a museum during a two-week trial. Experimental results revealed that visitors evaluated the robot with the GAC system higher than without it.

We believe that our findings will lead to the development of humanoid robots that can smoothly and naturally engage in group interactions. While these preliminary findings show promising usage of robot behavior in interactions between a robot and groups, one of our future goals remains to develop a completely autonomous mechanism for controlling group attention.

\section{ACKNOWLEDGMENTS}

We wish to thank the staff at the Osaka Science Museum for their kind cooperation. We also wish to thank the following ATR members for their helpful suggestions and cooperation: Hideaki Terauchi, Toshihiko Shibata, and Kenta Nohara. This research was supported by the Ministry of Internal Affairs and Communications of Japan.

\section{REFERENCES}

[1] Fujita, M. "AIBO: Toward the era of digital creatures," Int. J. Robot, Res., vol. 20, no. 10, pp. 781-794, 2001.

[2] Shibata, T. "An overview of human interactive robots for psychological enrichment," The Proceedings of IEEE, 2004.

[3] Ishiguro, H., Ono, T., Imai, M., Maeda, T., Kanda, T. and Nakatsu, R. "Robovie: an interactive humanoid robot," Int. J. Industrial Robot, Vol. 28, No. 6, pp. 498-503, 2001.

[4] Kozima, H., Nakagawa, C., Kosugi, D., Kawai, N., and Yano, Y. "A humanoid robot in company with children," IEEE RAS/RSJ Int. Conf. Humanoid Robotics, CD-ROM, 2004.

[5] Breazeal, C. and Scassellati, B. "How to build robots that make friends and influence people," IEEE/RSJ Int. Conf. Intelligent Robots and Systems, 1999.

[6] Kanda, T., Hirano, T., Eaton, D., and Ishiguro, H. "Interactive Robots as Social Partners and Peer Tutors for Children: A Field Trial," Human Computer Interaction, Vol. 19, No. 1-2, pp. 61-84, 2004.

[7] Siegwart, R. et al. "Robox at Expo. 02: A Large Scale Installation of Personal Robots," Robotics and Autonomous Systems, 42, pp. 203-222, 200.

[8] Shiomi, M., Kanda, T., Ishiguro, H., and Hagita, N. "Interactive Humanoid Robots for a Science Museum," 1st Annual Conference on Human-Robot Interaction, pp. 305312, 2006.

[9] Nourbakhsh, I., Bobenage, J., Grange, S., Lutz, R., Meyer, R., and Soto, A. "An Affective Mobile Educator with a Full-time Job," Artificial Intelligence, 114 (1-2), pp. 95-124, 1999.

[10] Woods, S., Walters, M., Lee Koay K., and Dautenhahn, K. "Comparing Human-Robot Interaction Scenarios Using Live and Video-Based Methods," Towards a Novel Methodological Approach, AMC'06, The 9th International Workshop on Advanced Motion Control.

[11] Green, A., Hüttenrauch, H., and Severinson Eklundh, K. "Applying the Wizard-of-Oz Framework to Cooperative Service Discovery and Configuration," 13th IEEE International Workshop on Robot and Human Interactive Communication, 2004.

[12] Horvitz, E., Jacobs, A., and Hovel, D. "Attention-Sensitive Alerting," The Fifteenth Conference on Uncertainty and Artificial Intelligence, pp. 305-313, 1998. 